OPEN ACCESS

Edited by:

Xiaolong $\mathrm{Li}$,

Indiana State University, United States

Reviewed by:

Bingliang Fang,

University of Texas MD Anderson

Cancer Center, United States

Olga Papadodima,

National Hellenic Research

Foundation, Greece

*Correspondence:

Kyle M. Schachtschneider

kschach2@uic.edu

Specialty section:

This article was submitted to

Systems Biology,

a section of the journal

Frontiers in Genetics

Received: 22 January 2021 Accepted: 07 April 2021

Published: 29 April 2021

Citation:

Patel SS, Sandur A, El-Kebir M,

Gaba RC, Schook LB and

Schachtschneider KM (2021)

Transcriptional Profiling of Porcine HCC Xenografts Provides Insights Into Tumor Cell Microenvironment

Signaling. Front. Genet. 12:657330.

doi: 10.3389/fgene.2021.657330

\section{Transcriptional Profiling of Porcine HCC Xenografts Provides Insights Into Tumor Cell Microenvironment Signaling}

\author{
Shovik S. Patel'1, Amitha Sandur ${ }^{2}$, Mohammed El-Kebir², Ron C. Gaba', \\ Lawrence B. Schook ${ }^{1,3,4}$ and Kyle M. Schachtschneider ${ }^{1,4,5 *}$
}

'Department of Radiology, University of Illinois at Chicago, Chicago, IL, United States, ${ }^{2}$ Department of Computer Science, University of Illinois at Urbana-Champaign, Urbana, IL, United States, ${ }^{3}$ Department of Animal Sciences, University of Illinois at Urbana-Champaign, Urbana, IL, United States, ${ }^{4}$ National Center for Supercomputing Applications, University of Illinois at Urbana-Champaign, Urbana, IL, United States, ${ }^{5}$ Department of Biochemistry and Molecular Genetics, University of Illinois at Chicago, Chicago, IL, United States

Hepatocellular carcinoma $(\mathrm{HCC})$ is the second leading cause of cancer-related death worldwide, representing the most common form of liver cancer. As HCC incidence and mortality continue to increase, there is a growing need for improved translational animal models to bridge the gap between basic HCC research and clinical practice to improve early detection and treatment strategies for this deadly disease. Recently the Oncopig cancer model-a novel transgenic swine model that recapitulates human cancer through Cre recombinase induced expression of $K R A S^{G 12 D}$ and $T P 53^{R 167 H}$ driver mutations - has been validated as a large animal translational model for human HCC. Due to the similar size, anatomy, physiology, immunology, genetics, and epigenetics between pigs and humans, the Oncopig has the potential to improve translation of novel diagnostic and therapeutic modalities into clinical practice. Recent studies have demonstrated the importance of tumor cells in shaping its surrounding microenvironment into one that is more proliferative, invasive, and metastatic; however, little is known about the impact of microenvironment signaling on HCC tumor biology and differential gene expression between HCC tumors and its tumor microenvironment (TME). In this study, transcriptional profiling was performed on Oncopig HCC xenograft tumors $(n=3)$ produced via subcutaneous injection of Oncopig HCC cells into severe combined immunodeficiency (SCID) mice. To differentiate between gene expression in the tumor and surrounding tumor microenvironment, RNA-seq reads originating from porcine (HCC tumor) and murine (microenvironment) cells were bioinformatically separated using Xenome. Principle component analysis (PCA) demonstrated clustering by group based on the expression of orthologous genes. Genes contributing to each principal component were extracted and subjected to functional analysis to identify alterations in pathway signaling between HCC cells and the microenvironment. Altered expression of genes associated with hepatic fibrosis deposition, immune response, 
and neo angiogenesis were observed. The results of this study provide insights into the interplay between $\mathrm{HCC}$ and microenvironment signaling in vivo, improving our understanding of the interplay between HCC tumor cells, the surrounding tumor microenvironment, and the impact on HCC development and progression.

Keywords: hepatocellular carcinoma, hepatic fibrosis, microenvironment, RNA sequencing, xenograft, porcine cancer model

\section{INTRODUCTION}

Solid tumors consists of a population of cancer cells in addition to a variety of resident and infiltrating host cells, secreted factors and extracellular matrix proteins, collectively known as the tumor microenvironment (TME). Crosstalk between tumor cells and the TME can lead to modulation of the TME, resulting in development of a beneficial microenvironment for tumor cells to grow and evade detection and killing by infiltrative immune cells (Whiteside, 2008).Tumor-derived signaling can not only allow tumor cells to escape the host immune system, but also promotes tumor cell growth. These interactions between cancer cells and the cellular and non-cellular components of the TME promote many aspects of tumor development, including cancer cell evolution, progression, and metastasis (Baghban et al., 2020). Understanding the underlying cellular and molecular mechanisms governing these interactions can inform development of novel therapeutic strategies designed to disrupt this signaling and restore anti-tumor activity (Whiteside, 2008).

Hepatocellular carcinoma (HCC) is the 5th most common cancer globally and the 2nd most common cause of cancerrelated death worldwide, accounting for $>9 \%$ of yearly cancer mortality (Flores and Marrero, 2014; Kirstein, 2016). HCC most commonly develops in cirrhotic liver microenvironments defined by increased fibrosis deposition and chronic inflammation, and there is growing evidence for the importance of cross-talk between tumor cells and the surrounding TME for modulating the progression of hepatocarcinogenesis, epithelial mesenchymal transitions, tumor invasion, and metastasis (Mhatre et al., 2012). HCC incidence in the United States has tripled over the past three decades due to the high incidence of liver cirrhosis from alcohol abuse and hepatitis $\mathrm{C}$ virus infection; the increasing prevalence of non-alcoholic steatohepatitis (NASH) due to the growing worldwide obesity epidemic ensures that HCC will continue to be an important public health concern in the future (Rahib et al., 2020). As HCC incidence and mortality continue to increase, there is a growing need for research into the impact of tumor cell-TME crosstalk to better understand HCC progression and develop novel treatment strategies for this deadly disease.

Advances in cancer research are reliant on the use of novel, clinically relevant preclinical cancer models. Recently the Oncopig Cancer Model-a novel transgenic swine model that recapitulates human cancer through Cre recombinase induced expression of $K R A S^{G 12 D}$ and $T P 53^{R 167 H}$ driver mutations (Schook et al., 2015) - has been validated as a large animal translational model for human HCC (Schachtschneider et al., 2017a; Gaba et al., 2020). The similar size, anatomy, physiology, immunology, metabolism, genetics, and epigenetics between pigs and humans allows for translation of results to clinical practice to improve diagnostics and therapeutic applications (Schachtschneider et al., 2017b). This model can also provide insights into the molecular mechanisms involved in tumor development, signaling involved in development and maturation of the TME, and subsequent host responses towards cancer development.

The purpose of this study was to investigate HCC-TME crosstalk to better understand the HCC tumor cell and TMEderived mechanisms leading to development and modulation of the HCC TME. In order to distinguish between HCC tumor cell and TME signaling, RNA-seq was performed on Oncopig HCC xenograft tumors produced via injection of Oncopig HCC cells into SCID mice. To differentiate between gene expression in the tumor and surrounding tumor microenvironment, RNAseq reads originating from porcine (tumor) and murine (TME) cells were separated bioinformatically to allow for individual quantification of HCC and TME-specific gene expression. The results of this study provide insights into the mechanisms through which HCC cells and the TME work in concert to modulate the TME and promote HCC proliferation.

\section{METHODS}

\section{Ethics Statement:}

Animal studies and tissue collection was conducted at The University of Illinois at Urbana-Champaign in accordance with national and international guidelines and approved by The University of Illinois Institutional Animal Care and Use Committee (IACUC protocol numbers 10189 and 10163).

\section{Oncopig HCC Xenograft Induction and Collection:}

Primary hepatocytes were isolated and transformed into HCC cells from Oncopig liver tissues ( $n=2$ female, $n=1$ male) as previously described (Schachtschneider et al., 2017a; Gaba et al., 2020). Briefly, primary hepatocytes were isolated from resected Oncopig liver tissues. Twenty-four hours post isolation, primary hepatocytes were incubated with an adenoviral vector encoding Cre recominase, resulting in transgene expression and subsequent transformation into HCC cell lines. The 3 distinct Oncopig HCC cell lines were cultured for 3 weeks before injection into female SCID mice (NOD.CB17-Prkdcscid/JAX, Bar Harbor, ME, United States). For xenograft tumor generation, $1 \times 10^{7}$ Oncopig HCC cells suspended in $100 \mu \mathrm{L}$ phosphate buffered saline were subcutaneously injected into each mouse $(n=3)$. 
21 days post injection, mice were euthanized, and tumors were harvested. Half of the tumor samples were flash frozen in liquid nitrogen and stored at -80 degrees Celsius until further processing. The other half was formalin fixed for histological confirmation of HCC.

\section{RNA Isolation:}

Total RNA was extracted from the tumor samples using the AllPrep DNA/RNA Mini Kit (Qiagen, Valencia, CA, United States) following the manufacturer's protocol. RNA integrity and confirmation of a lack of genomic DNA contamination was determined using an Agilent 2100 Bioanalyzer using an RNA Nano bioanalyzer chip by the Carver High-Throughput DNA Sequencing and Genotyping Unit (HTS lab, University of Illinois, Urbana, IL, United States). RNA samples were utilized for sequencing if their RNA integrity number was greater than 7 .

\section{RNA-seq Library Preparation:}

TruSeq Stranded RNA-seq libraries (TruSeq Stranded RNA Sample Preparation Kit, Illumina, San Diego, CA, United States) were produced from high-quality RNA (1 $\mu \mathrm{g})$ by the HTS lab (University of Illinois, Urbana, IL, United States) following standard protocols. RNA-seq libraries were paired-end sequenced $(2 \times 100 \mathrm{bp})$ on an Illumina HiSeq2000. All datasets are available in the NCBI Short Read Archive under accession number PRJNA685801.

\section{Species-Based Read Separation and Mapping to Reference Genomes:}

An average of 29 million raw stranded paired-end reads were produced for each sample, ranging from 28.4 to 29.4 million. Trim Galore version $0.4 .4^{1}$ was used to trim raw reads for adapter contamination. Quality Phred score cutoff was set to 30 , maximum trimming error rate was set to 0.1 , and minimum sequence length was set to 20 with a stringency of 6 . Reference genomes and transcriptomes were obtained from the Ensembl genome database. Mus_musculus.GRCm38.99 was used as the reference for mouse, and Sus_scrofa.Sscrofa11.1.99 (Warr et al., 2020) was used as the reference for pig. Xenome Version 1.0.0 from the Gossamer bioinformatics suite was used to separate reads between mouse and pig using default settings (Conway et al., 2012). Mouse was set as the host genome while pig was set as the graft genome. Reads were classified into the following classifications: host, graft, ambiguous, both, and neither. STAR 2.7.3a was used to map output reads from Xenome (Dobin et al., 2013). Only host and graft classified reads from Xenome were used for STAR alignment to the species-specific genome and transcriptome. The following non-default options were used for mapping with STAR: -sjdbOverhang 99, -outFilterType BySJout, -outFilterMultipmapNmax 20,-alignSJoverhangMin 8, -alignSJDBoverhangMin 1, -outFilterMismatchNmax 999, -outFilterMismatchNoverReadLmax 0.04, outSAMtype BAM SortedByCoordinate. A second pass mapping was performed

${ }^{1}$ https://github.com/FelixKrueger/TrimGalore using SJ.out.tab files from the first pass. RSEM version 1.3.1 was used to quantify gene expression (Li and Dewey, 2011). The following options were used with the rsem-calculateexpression function: -strandedness reverse, -alignments, paired-end.

\section{Cross Species Analysis:}

RStudio Version 1.2.5042 was used for data filtering and analysis of the RSEM gene quantifications. A list of known one-toone orthologous genes between mouse and pig was created through Ensembl's Biomart database. Genes were filtered to retain only one-to-one orthologues expressed in at least 1 sample. Transcripts per million (TPM) gene expression values were normalized through addition of a.01 pseudocount to all expression values followed by $\log$ base 2 transformation as previously described for comparison of gene expression across species (Foissac et al., 2019). Principle Component Analysis (PCA) plots were produced using the factoextra() package in RStudio Version 1.2.5042 using the $\operatorname{prcomp}()$ function. A list of genes that contributed to principle components 1 and 2 was extracted from the PCA. From these lists, all genes that were above the expected average contribution value were used for pathway analysis.

\section{Pathway Analysis:}

The list of genes contributing to principle component 1 and 2 above the expected average contribution value were analyzed using Ingenuity Pathway Analysis (IPA) (QIAGEN Inc., Krämer et al., 2014) ${ }^{2}$. Average TPM values for each species were determined, and $\log 2$ fold changes were calculated between the average mouse and average pig TPM values. The following equation was used: Log2 fold change $=\log 2$ (Average Mouse TPM/Average Pig TPM). Based on this calculation, a negative $\log 2$ fold change value indicates that the gene displays higher expression in the HCC tumor while a positive $\log 2$ fold change value indicates that the gene displays higher expression in the Tumor Microenvironment (TME). Differentially expressed genes (DEGs) between the TME (murine genes) and the HCC tumor (porcine genes) were assigned as those that had log2 fold change values of $\leq-2.2$ or $\geq+2.2$. These values were selected to help ensure comparisons were based on biologically relevant differences in gene expression, as well as to allow for performance of pathway analysis using IPA's recommended input range (200 to 3,500 genes). The principle component 1 and 2 gene lists alongside its corresponding log 2 fold change value was uploaded for IPA's core analysis (Supplementary Tables 1,2). Core analysis revealed significantly enriched groups of genes associated with canonical pathways and diseases \& functions derived from IPA's database. $Z$-scores were calculated for each canonical pathway. The $Z$-scores reflect predicted activation or inhibition states of canonical pathways based off the expression of available genes in our dataset. With respect to our dataset, positive Z-scores indicate activation in the murine TME while negative $Z$-scores indicate activation in the porcine HCC tumors. $P$-values obtained from IPA are calculated using a Right-Tailed Fisher's Exact

\footnotetext{
${ }^{2}$ https://www.qiagenbioinformatics.com/products/ingenuitypathway-analysis
} 
Test. These $P$-values were then corrected for multiple testing using the Benjamin-Hochberg false discovery rate (FDR), using a FDR value of 0.05 .

\section{RESULTS}

\section{Species-Based Read Separation:}

Reads originating from mouse (TME) and pig (HCC) cells were successfully separated bioinformatically and assigned to their respective reference genomes (Table 1). The majority of reads (>93\%) were either classified as Host (mouse, TME) or Graft (pig, HCC) in all samples. The average percentage of reads derived from the TME and HCC tumor cells was 52.2 and $42.3 \%$, respectively (Table 1). Once separated, species-specific reads were aligned to their respective reference genomes (Supplementary Table 3). On average, mouse reads uniquely mapped to the mouse genome at a rate of $90.13 \%$, while pig reads uniquely mapped to the pig genome at a rate of $86.08 \%$. Given the ability to successfully separate and align reads based on species of origin, further analyses were performed to investigate HCC tumor and TME signaling patterns.

\section{Genome-Wide Cross-Species Expression Analysis:}

Gene expression levels were quantified for the 55,471 and 31,907 genes annotated in the mouse and pig genome, respectively. Of the 24,011 one-to-one orthologues identified between mice and pigs, 14,163 were expressed in at least one sample and used for downstream analyses. PCA and hierarchical clustering analysis based on the 14,163 one-to-one orthologues expressed in at least one sample resulted in samples clustering by group (Figures 1A,B). Principle component 1 represents $61 \%$ of the variation in the dataset and differentiates between the HCC tumor and TME samples. Interestingly, principle component 2, which represents $17.6 \%$ of the variation, differentiates between individual HCC tumor/TME sample pairs. In total 8,060 genes were identified to significantly contribute to principle component 1 (Figure 1C), while 4,603 genes significantly contributed to principle component 2 (Figure 1D).

\section{Pathway Enrichment Analysis:}

In order to investigate the biological relevance of the differential gene expression observed between HCC tumor and TME samples, genes that contributed to each principle component at a higher rate than expected by chance were extracted for pathway analysis. As differential expression analysis was performed between HCC cells and subcutaneous TME cells (i.e., fibroblasts, inflammatory cells, vascular tissue), some of the differential expression observed may be due to differences in baseline expression between these cell and tissue types. Therefore, genes utilized for pathway analysis were further limited to those with a $\log 2$ fold change in expression of $\leq-2.2$ or $\geq+2.2$ to help ensure comparisons were based on biologically relevant differences in gene expression while reducing noise related to baseline expression differences between cell types. The final number of DEGs used for pathway analysis was 3,347 for principal component 1 (1,424 genes upregulated in the HCC tumor and 1,923 genes upregulated in the TME) and 269 genes for component 2 (114 genes upregulated in the HCC tumor and 155 genes upregulated in the TME). Functional enrichment analysis of principal component 1 genes resulted in identification of pathways involved in hepatic fibrosis, immune system regulation, inflammation regulation, and neo angiogenesis (Table 2). In addition, the top diseases and disorders enriched for genes from principal component 1 included cancer, organismal injury and abnormalities, and gastrointestinal disease (Supplementary Table 4), while the top enriched molecular and cellular functions included cellular movement and cell-to-cell signaling interaction (Supplementary Table 5). Finally, the top physiological system development and functions enriched for genes from principal component 1 included immune cell trafficking (Supplementary Table 6). Pathway analysis on principle component 2 did not reveal enrichment of any canonical pathways. However, top diseases and disorders enriched for genes from principal component 2 included cancer, endocrine system disorders, and gastrointestinal disease (Supplementary Table 7).

\section{Hepatic Fibrosis Induced by Signaling Between HCC Tumor and Its Microenvironment:}

Pathway analysis revealed enrichment of two liver specific pathways involved in fibrosis: hepatic stellate cell (HSC) activation pathway and hepatic fibrosis signaling pathway, with the HSC activation pathway representing the most significantly enriched pathway $(-\log (p$-value $)=14.7$; Table 2$)$. The hepatic fibrosis signaling pathway had a $Z$-score greater than 2 indicating that this pathway is significantly upregulated in the TME.

TABLE 1 | Read Classifications.

\begin{tabular}{|c|c|c|c|c|c|}
\hline & \% "Host" Reads & $\%$ "Graft" Reads & \% "Ambiguous" Reads & \% "Both" Reads & $\%$ "Neither" Reads \\
\hline Tumor 42 & $39.50 \%$ & $54.10 \%$ & $3.50 \%$ & $0.97 \%$ & $1.90 \%$ \\
\hline Average & $52.2 \%$ & $42.3 \%$ & $3.1 \%$ & $0.87 \%$ & $1.51 \%$ \\
\hline
\end{tabular}

Proportion of reads Xenome classified to each category for all samples. The category "Host" refers to reads classified to the mouse reference genome while "Graft" refers to reads classified to the pig reference genome. The category "Both" refers to reads that match both mouse and pig reference genomes, "neither" represents reads that do not match either reference genome, and "Ambiguous" represents reads that are likely artifacts created during library preparation. 


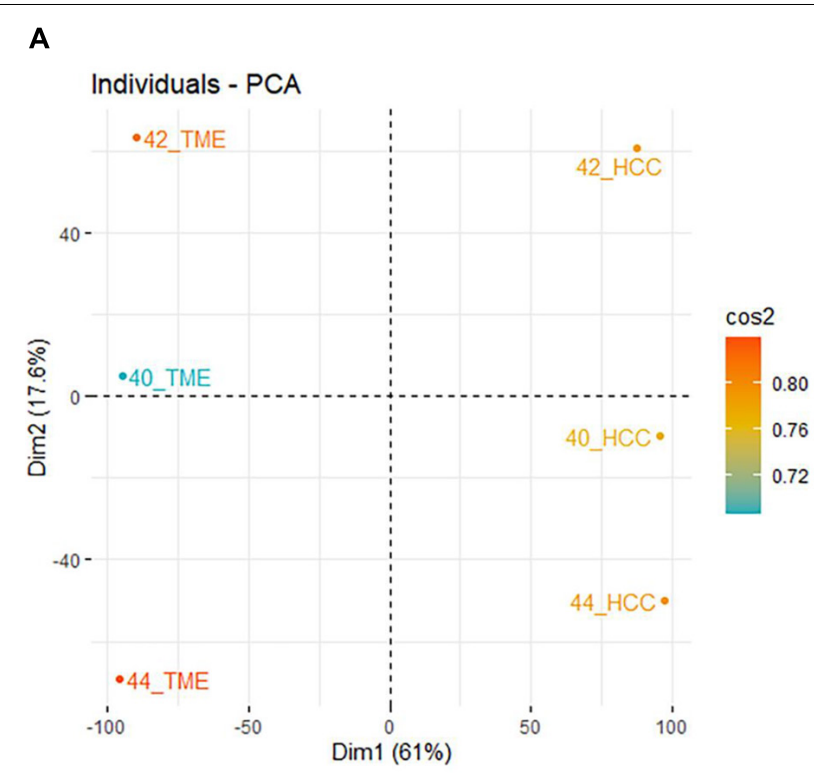

C Contribution of variables to Dim-1

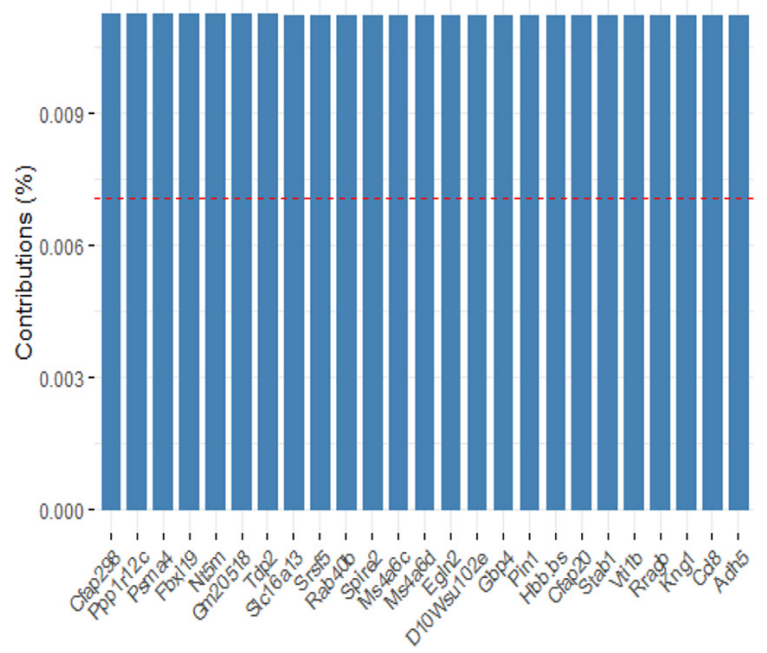

B

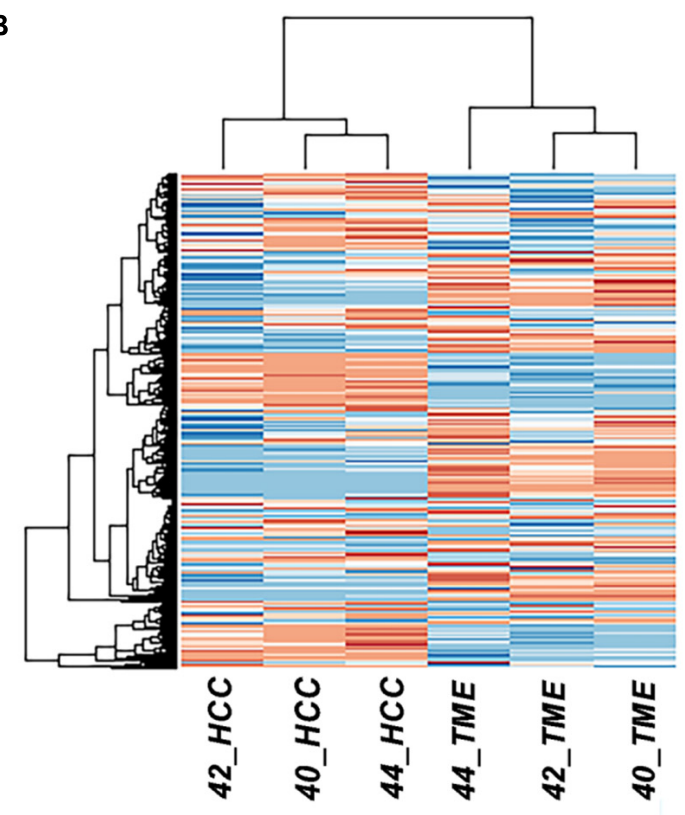

D Contribution of variables to Dim-2

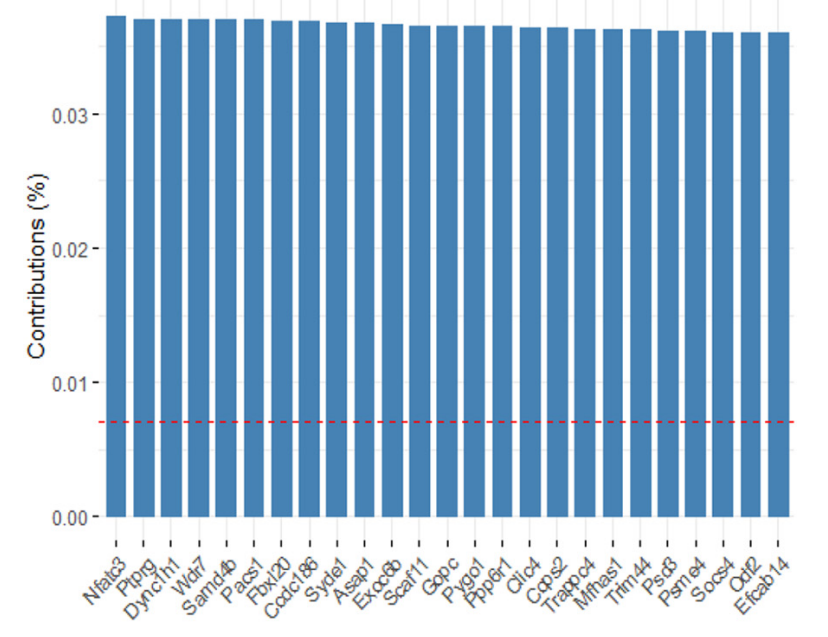

FIGURE 1 | Differential Expression Between HCC Tumors and the TME. (A) PCA plot and (B) Heatmap based on the final filtered list of 14,163 orthologous genes expressed in at least 1 sample. (C) Top 25 genes contributing to the variation of dimension 1. (D) Top 25 genes contributing to the variation of dimension 2.

A total of 69 genes in the HSC Activation Pathway were differentially expressed in this study, 53 and 16 of which displayed higher expression in the TME and HCC tumors, respectively (Figure 2A). In addition, a total of 82 genes in the Hepatic Fibrosis Signaling pathway were altered in this study, 52 and 29 of which displayed higher expression in the TME and HCC tumors, respectively (Figure $2 \mathrm{~B}$ ).

A key molecular and cellular driver in the progression of liver fibrosis is the activation of HSCs. In normal physiological liver function, quiescent HSCs act as a storage for Vitamin A. Expression of inflammatory molecules and mediators following acute liver injury causes HSC activation and transdifferentiation into highly proliferative, pro-inflammatory, and migratory myofibroblasts that produce collagen and many other extracellular matrix (ECM) proteins (Puche et al., 2013; Zhang et al., 2016; Higashi et al., 2017). In this study, myofibroblast activation was observed as evidenced by increased ACTA2 in the TME ( $\log 2$ fold change $=6.52)$. ACTA2 is a proven myofibroblast marker, and is highly expressed in myofibroblasts irrespective of their precursor cell type (Watsky et al., 2010). The presence of myofibroblasts in the TME was likely driven by increased expression of TGFB (log2 fold change $=2.78$ ) and its receptor TGFBR3 $(\log 2$ fold change $=6.29)$ in the TME, as TGFB is one of the most potent activators of myofibroblast differentiation 
TABLE 2 | Top 20 Pathways enriched for genes significantly contributing to principal component 1.

\begin{tabular}{|c|c|c|c|c|c|c|}
\hline Ingenuity Canonical Pathways & $\begin{array}{l}\text { adjusted } \\
p \text {-value }\end{array}$ & Ratio & z-score & Total genes & $\begin{array}{c}\text { \# of genes } \\
\text { upregulated } \\
\text { in HCC tumor }\end{array}$ & $\begin{array}{c}\text { \# of genes } \\
\text { upregualted } \\
\text { in TME }\end{array}$ \\
\hline 1. Hepatic Fibrosis/Hepatic Stellate Cell Activation & $4.11 \mathrm{E}-13$ & 0.371 & undetermined & 69 & 16 & 53 \\
\hline 2. Axonal Guidance Signaling & $1.3 \mathrm{E}-11$ & 0.264 & undetermined & 128 & 50 & 78 \\
\hline 3. Leukocyte Extravasation Signaling & 4.33E-11 & 0.34 & 1.192 & 67 & 26 & 41 \\
\hline 4. Atherosclerosis Signaling & $2.05 E-10$ & 0.386 & undetermined & 49 & 21 & 28 \\
\hline 5. Granulocyte Adhesion and Diapedesis & $2.73 E-10$ & 0.339 & undetermined & 61 & 30 & 31 \\
\hline 6. LXR/RXR Activation & $2.73 E-10$ & 0.388 & -3.618 & 47 & 27 & 20 \\
\hline 7. FXR/RXR Activation & 5E-09 & 0.365 & undetermined & 46 & 36 & 8 \\
\hline 8. Coagulation System & $7.78 \mathrm{E}-09$ & 0.6 & -0.218 & 21 & 16 & 5 \\
\hline 9. Agranulocyte Adhesion and Diapedesis & $1.35 \mathrm{E}-08$ & 0.311 & undetermined & 43 & 20 & 23 \\
\hline 10. GP6 Signaling Pathway & $1.83 \mathrm{E}-08$ & 0.361 & 4.727 & 43 & 7 & 36 \\
\hline 11. Breast Cancer Regulation by Stathmin1 & $1.83 \mathrm{E}-08$ & 0.23 & undetermined & 136 & 48 & 88 \\
\hline $\begin{array}{l}\text { 12. Role of Macrophages, Fibroblasts and } \\
\text { Endothelial Cells in Rheumatoid Arthritis }\end{array}$ & $7.85 \mathrm{E}-08$ & 0.263 & undetermined & 82 & 26 & 56 \\
\hline 13. Cardiac Hypertrophy Signaling (Enhanced) & $2.51 \mathrm{E}-07$ & 0.232 & 2.83 & 113 & 44 & 69 \\
\hline $\begin{array}{l}\text { 14. Role of Osteoblasts, Osteoclasts and } \\
\text { Chondrocytes in Rheumatoid Arthritis }\end{array}$ & $7.72 \mathrm{E}-07$ & 0.277 & undetermined & 61 & 25 & 36 \\
\hline 15. Sperm Motility & $1.22 \mathrm{E}-06$ & 0.274 & 2.2 & 61 & 21 & 40 \\
\hline $\begin{array}{l}\text { 16. Role of Pattern Recognition Receptors in } \\
\text { Recognition of Bacteria and Viruses }\end{array}$ & $6.97 \mathrm{E}-06$ & 0.292 & 2.6 & 45 & 13 & 32 \\
\hline 17. Hepatic Fibrosis Signaling Pathway & $6.97 \mathrm{E}-06$ & 0.234 & 2.871 & 82 & 29 & 53 \\
\hline 18. Calcium Signaling & $9.84 \mathrm{E}-06$ & 0.267 & 2.271 & 55 & 17 & 38 \\
\hline 19. Colorectal Cancer Metastasis Signaling & $9.84 \mathrm{E}-06$ & 0.253 & 2.405 & 64 & 25 & 39 \\
\hline 20. Synaptogenesis Signaling Pathway & $9.84 \mathrm{E}-06$ & 0.24 & 1.65 & 75 & 31 & 44 \\
\hline
\end{tabular}

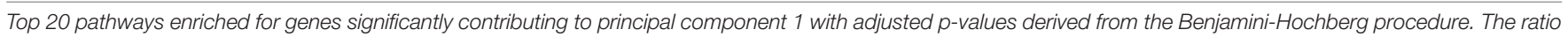

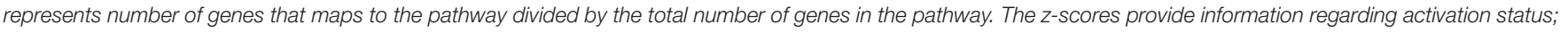
$z$-scores $>2$ predict activation of the pathway in the TME while $z$-scores $<-2$ predicts activation of the pathway in the HCC tumor.

and has been shown to induce ACTA2 expression (Harris et al., 2013; Breton et al., 2018). Additionally, IPA revealed ACTA2 upregulation via TGFB mediated regulation (Figure 3). While these results demonstrate that TME cells represent the source of $T G F B$ secretion, $T G F B$ must be activated from its latent form in order to induce myofibroblast differentiation. Key TGFB activators include integrin proteins (Goodwin and Jenkins, 2009; Khan and Marshall, 2016), 4 of which (ITGA2, ITGA3, ITGB1, ITGB3) displayed increased expression in the HCC tumor cells ( $\log 2$ fold change $=-6.16,-5.56$, -2.32 , and -4.35 , respectively). Additionally, IPA revealed an interaction between CCN2 and integrins that could lead to inhibition of cell cycle arrest and HSC proliferation through $A K T$ signaling (Figure 3). Increased CCN2 expression (log2 fold change $=-4.10$ ) was observed in the HCC tumor cells and is associated with TGFB-mediated fibrosis deposition (Nakerakanti et al., 2011).

In addition to increased ACTA2 expression, 21 type I and type III collagen genes showed increased expression in the TME (Figures 3, 4). Only one collagen gene, COL17A1 ( $\log 2$ fold change $=-8.43$ ), was found to be upregulated in the HCC tumor. In addition, increased expression of genes promoting collagen production was observed in both the HCC tumor and TME cells. In the TME, increased TGFB expression promotes type I and type III collagen production (Figures 3,4 ), while increased IGF1 expression $(\log 2$ fold change $=6.55)$ promotes production of type 1 collagen (Figure 4). In addition, increased expression of TGFA ( $\log 2$ fold change $=-2.28$ ), a potent mitogen for hepatocytes that is associated with hepatocarcinogenesis (Harada et al., 1999; Yeh et al., 2007), was observed in HCC cells resulting in upregulation of type I collagen (Figure 4).

ECM remodeling proteins were also differentially expressed in this study. Matrix metalloproteinases (MMPs) and tissue inhibitors of metalloproteinases (TIMPs) are major players in ECM remodeling and play a role in HCC progression and invasion (Scheau et al., 2019). In this study, multiple MMPs and TIMPs displayed altered expression between the HCC tumor and TME. MMP2 (log2 fold change = 7.47), MMP9 $(\log 2$ fold change $=3.63)$, and TIMP2 $(\log 2$ fold change $=2.66)$ displayed increased expression in the TME, while MMP13 $(\log 2$ fold change $=-4.41)$ and TIMP1 $(\log 2$ fold change $=-2.26)$ displayed increased expression in the HCC tumor. In addition, increased expression of several genes in the TME known to drive expression of HCC tumor-derived TIMP1 and MMP13 was observed (Figures 3, 4). These factors include TGFB and TNF ( $\log 2$ fold change $=2.78$ and 4.38 , respectively). IPA revealed TGFB mediated upregulation of MMP13 (Figure 4A) and TNF mediated upregulation of $M M P 13$ (Figure 3). Likewise, increased expression of IL1A (log2 fold change $=-4.15)$ and TGFA $(\log 2$ fold change $=-2.28$ ) was observed in the HCC tumor cells 


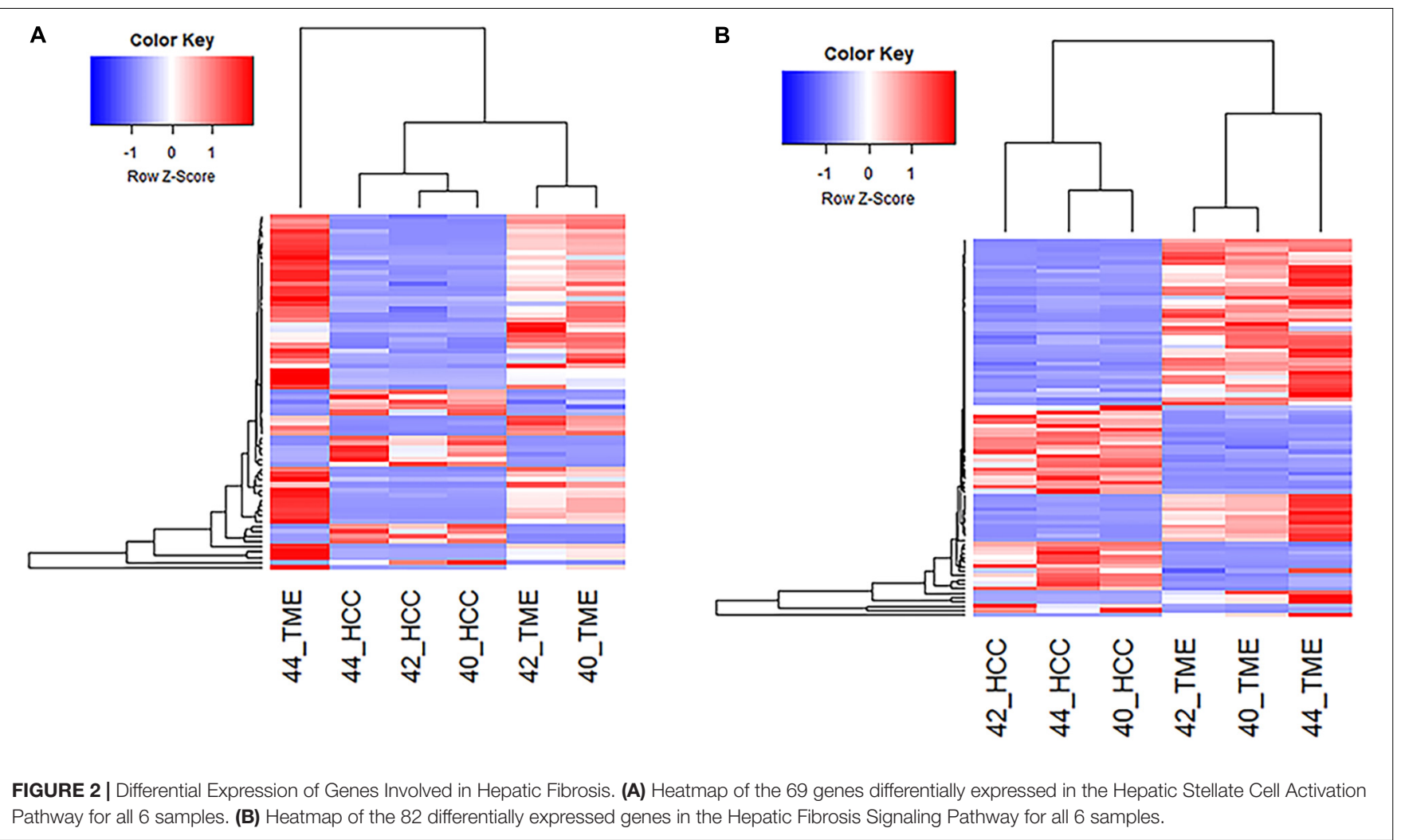

leading to increased expression of TME-derived MMP9 and $M M P 2$, respectively (Figure 4).

\section{Innate Immune System and Inflammatory Signals Derived From HCC Cells:}

In our dataset, pathway analysis revealed enrichment of multiple pathways associated with innate immune surveillance and chronic inflammation (Table 2). These pathways include the previously described hepatic fibrosis pathways, and NF- $\kappa B$ signaling $(-\log (p$-value $)=5.81, Z$-score $=2.598)$. The NF- $\kappa \mathrm{B}$ signaling pathway is activated in the TME and contains 49 DEGs, 35 of which displayed higher expression in the TME and 14 with higher expression in the HCC tumors (Figure 5).

With respect to the hepatic fibrosis pathways, DEGs of interest include TNF, TNFRSF1B, and $L B P$. TNF displayed higher expression in the TME ( $\log 2$ fold change $=4.38$ ), while its binding receptor TNFRSF1B displayed higher expression in the HCC tumor ( $\log 2$ fold change $=-2.25$ ). TNF is a major inflammatory cytokine produced mainly by macrophages and is a key contributor to many malignancies including HCC (Liu et al., 2013; Tan et al., 2019). In our dataset, TME-derived TNF appears to interact with HCC tumor cells expressing TNFRSF1B to induce upregulation of HCC-derived CCN2 $(\log 2$ fold change $=-4.10)$, MIP2 (log2 fold change $=-6.14)$, and VCAM1 $(\log 2$ fold change $=-2.46$; Figures 3, 4A). CCN2 is a secreted growth factor that interacts with various ECM molecules and is known to promote HCC progression through increased proliferation of cancer-associated fibroblasts and increased migration of macrophages (Mazzocca et al., 2010; Akahoshi et al., 2016; Makino et al., 2018). MIP2 is a cytokine that recruits neutrophils in the setting of liver injury (Moles et al., 2014; Qin et al., 2017), while VCAM1 expression during inflammatory diseases provides a scaffold for leukocyte migration and infiltration (Cook-Mills et al., 2011).

In the pathogenesis of liver injury, lipopolysaccharides from bacteria play a role as potent mediators of hepatic inflammation and fibrosis. $L B P$ encodes a protein that binds to bacterial lipopolysaccharides, eliciting an immune response (Gandhi, 2020). In our dataset, $L B P(\log 2$ fold change $=2.67)$ displayed higher expression in the TME. Myofibroblasts bind to LBPlipopolysaccharide complexes to induce expression of HCC tumor expressed MCP1 $(\log 2$ fold change $=-7.42)$ and VCAM1 $(\log 2$ fold change $=-2.46$; Figure $4 B)$, which displayed increased expression in the HCC tumors in this study. MCP1 is an inflammatory chemokine that is crucial for monocyte infiltration into tissues during inflammation (Germain, 2017). VCAM1 is a protein capable of mediating adhesion of lymphocytes to the vascular endothelium which include increased positive expression in inflamed liver sinusoids (Osborn et al., 1989; Steinhoff et al., 1993; Ho et al., 2004).

$\mathrm{NF}-\mathrm{\kappa B}$ is a key transcriptional regulator of inflammatory responses and plays a prominent role in chronic liver diseases (Luedde and Schwabe, 2011). In this study, the NF- $\kappa B$ signaling pathway was significantly activated in the TME $(Z$-score $=2.6)$. Activation of NF- $\kappa \mathrm{B}$ leads to expression of pro-inflammatory genes and regulates the survival and activation of innate immune cells (Liu et al., 2017). DEGs identified in this pathway 


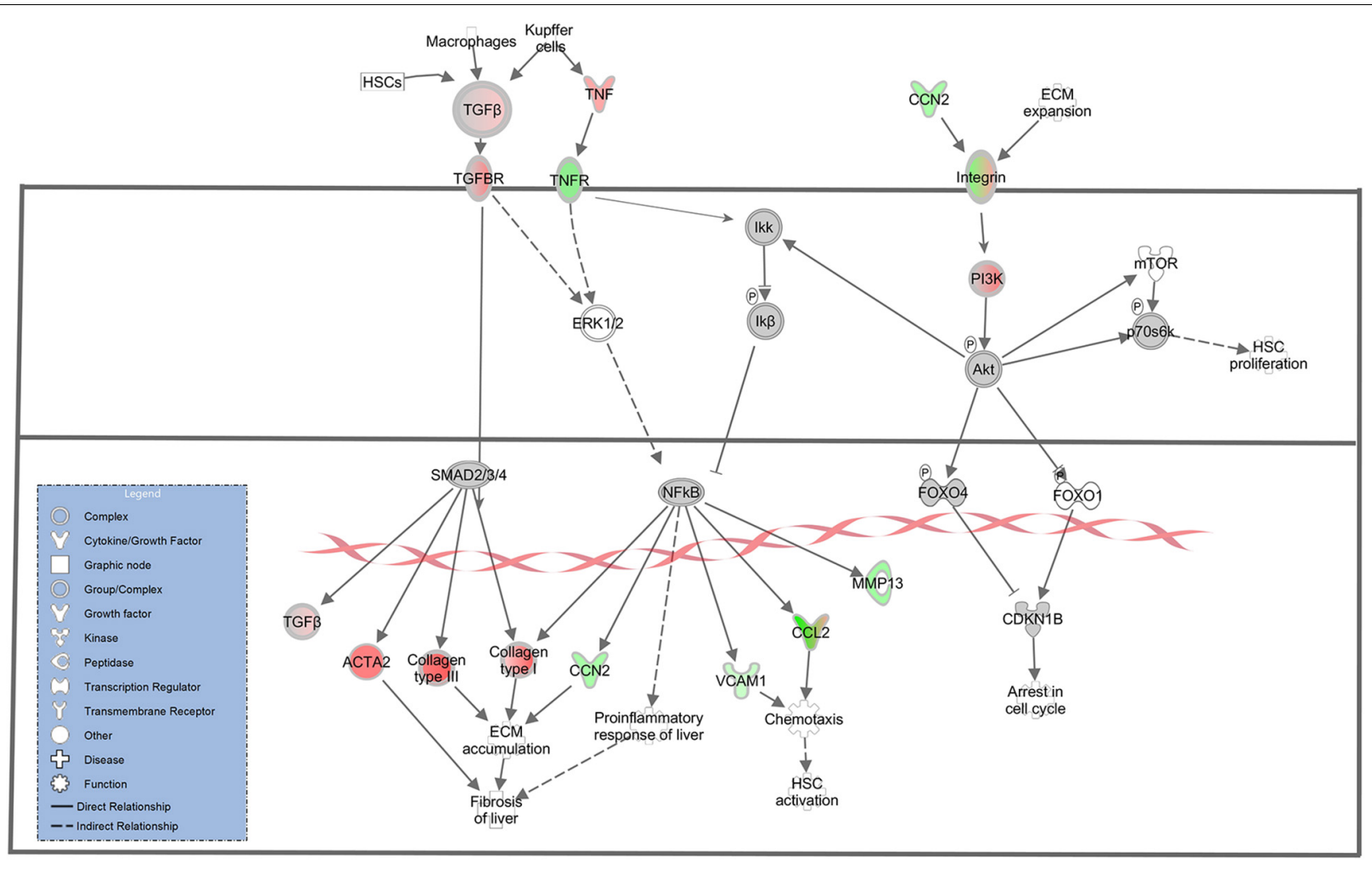

FIGURE 3 | Differential Expression in the Hepatic Fibrosis Signaling Pathway. Differential expression of genes in the hepatic fibrosis signaling canonical pathway in IPA (edited for simpler visualization; for full pathway, see Supplementary Figure 1). Molecule interactions are shown as explained in the legend. DEGs are highlighted either in red (upregulated TME genes) or green (upregulated HCC tumor genes). Color intensity indicates Increasing or decreasing degree of fold change.

include IL1A, TNF, TGFA, and RANKL. RANKL (log2 fold change $=7.39)$ is a potent stimulator of NF- $\mathrm{B}$ and its expression is associated with tumor migration and invasion in $\mathrm{HCC}$ (Song et al., 2014). It is upregulated in the TME and may induce NF- $\kappa \mathrm{B}$ signaling via binding to one of the HCC tumor expressed receptors, such as TNFRSF1B (Figure 5; $\log 2$ fold change $=-2.24) . I L 1 A(\log 2$ fold change $=-4.15)$ is upregulated in the HCC tumors and is likely signaling through IL1R2 ( $\log 2$ fold change $=4.74$ ) which is expressed in the TME. IL1R2 is a decoy receptor that IL1A can bind in order to prevent IL1A mediated signal transduction and may play protective roles in chronic inflammatory diseases like ulcerative colitis (Mora-Buch et al., 2016) and liver cirrhosis. This suggests that the TME could be responding to chemokines being produced by HCC tumors in order to limit an overactive inflammatory and immune response. As a consequence, the HCC tumors would be primed to grow in the immunotolerant microenvironment.

\section{Neo Angiogenesis Induced by HCC Tumor Signaling:}

In this study, two pathways related to neo angiogenesis were enriched for DEGs: the HIF1 $\alpha$ signaling pathway and the VEGF family ligand-receptor interactions pathway. The HIF1 $\alpha$ signaling pathway was activated in the TME $(-\log (p$-value $)=4.74 ; Z$-score $=2.1)$ and contains 51 DEGs,
31 and 20 of which display higher expression in the TME and HCC tumors, respectively (Figure 6). The VEGF Family Ligand-Receptor Interactions pathway $(-\log (p$-value $)=2$; $Z$-score $=1.41)$ is likely activated in the TME and contains 20 DEGs, 14 and 6 of which display higher expression in the TME and HCC tumors, respectively (Figure 6).

Hifl $\alpha$ is an important promoter of angiogenesis during hypoxic conditions. As HCC tumors are one of the most hypoxic tumors (Chen and Lou, 2017), Hifl $\alpha$ signaling is likely to be involved in HCC neovascularization. In our dataset, DEGs in the HIF1 $\alpha$ signaling pathway and VEGF family ligand-receptor interactions pathway that contribute to neo angiogenic growth include various vascular endothelial growth factors (VEGFRs), IGF1, EGF, ARNT, PAI1, and TGFA. Pathway analysis revealed two TME-derived growth factors, IGF1 ( $\log 2$ fold change $=6.55)$ and EGF ( $\log 2$ fold change $=5.24$ ) that are driving Hifl $\alpha$ signaling within the HCC tumor cells (Figure 6). This is evidenced by increased expression of HCC tumor-derived genes downstream of the Hifl $\alpha-A R N T$ complex (Figure 6). HCC tumor-derived ARNT ( $\log 2$ fold change $=-2.28)$ is a crucial gene involved in tumor progression that binds to Hifl $\alpha$ during hypoxic conditions to upregulate genes that contribute to invasion, metastasis, and neo angiogenesis (Tanimoto, 2000; Weir et al., 2011). From the data, the Hif $1 \alpha$-ARNT complex induces expression of proangiogenic factors including VEGFRs, PAI1 ( $\log 2$ fold change $=-4.08)$, and 


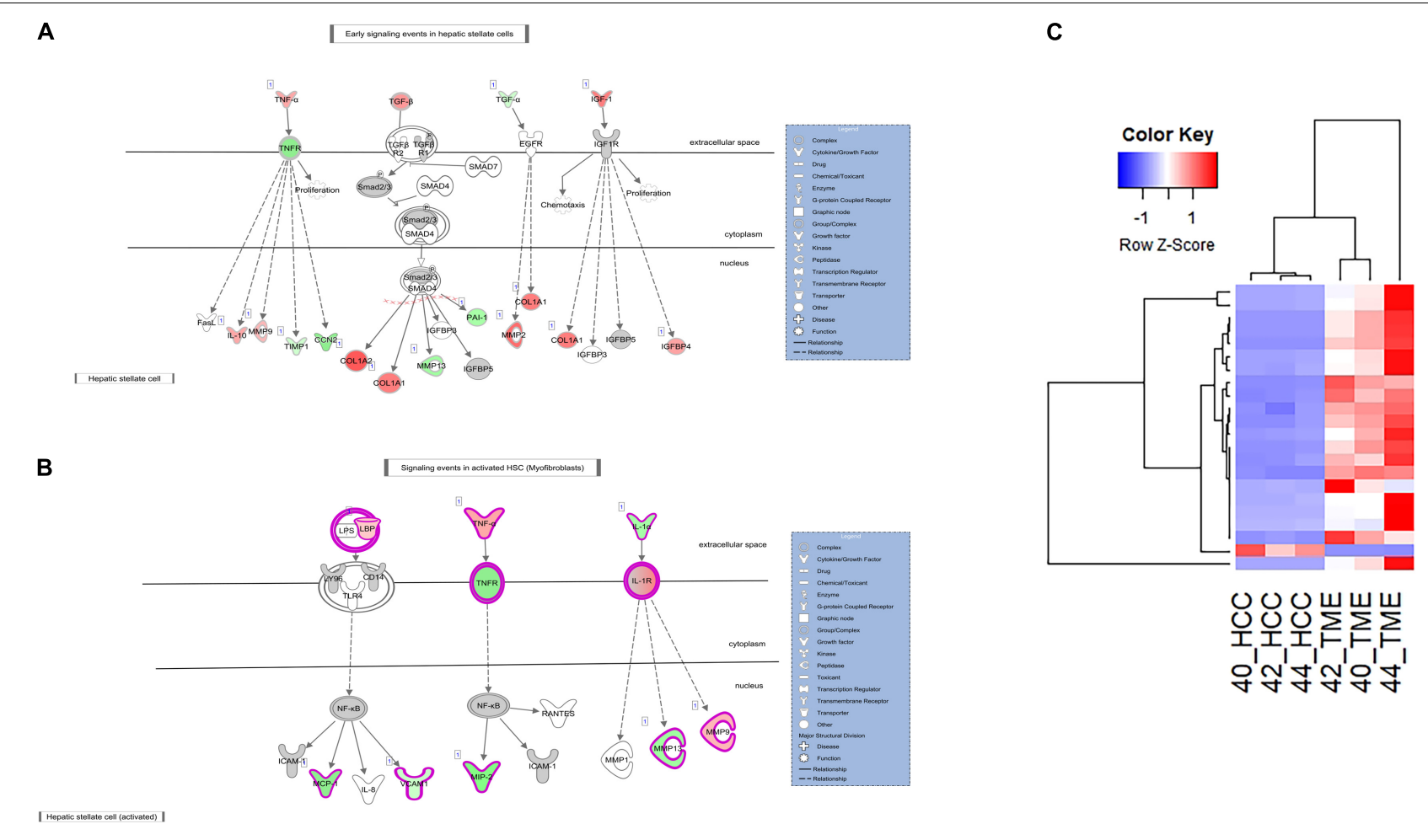

FIGURE 4 | Differential Expression in the Hepatic Stellate Cell Activation Pathway. (A,B) The hepatic stellate cell activation canonical pathway in IPA (edited for simpler visualization; for full pathway, see Supplementary Figure 2). Molecule interactions are shown as explained in the legend. DEGS are highlighted either in red (upregulated TME genes) or green (upregulated HCC tumor genes). Color intensity indicates increasing or decreasing degree of fold change. (C) Heatmap of the 22 collagen genes differentially expressed in the Hepatic Stellate Cell Activation Pathway for all 6 samples.

TGFA ( $\log 2$ fold change $=-2.27$ ). Additionally in the VEGF family ligand-receptor interactions pathway, VEGFR1 $(\log 2$ fold change $=-2.38$ ) was upregulated in the HCC tumor while NRP1 $(\log 2$ fold change $=4.93)$, VEGFR2 $(\log 2$ fold change $=2.76)$, and VEGFR3 ( $\log 2$ fold change $=3.21$ ) were upregulated in the TME.

\section{DISCUSSION}

In this study, we sought to examine differential gene expression between HCC tumor cells and TME cells by looking at genes expressed between porcine HCC tumor cells xenografted into SCID mice via RNA-seq and pathway analysis with IPA. Separating porcine HCC tumor and murine TME RNA-seq reads revealed a greater understanding of signaling pathway alterations and cross-talk between HCC tumor cells and its TME. The main signaling interactions observed between HCC tumors and their TME were related to hepatic fibrosis, inflammation, and neo angiogenesis mechanisms.

HCC typically develops in cirrhotic livers following hepatic fibrosis deposition; however, few studies have investigated how interactions between HCC tumor cells and TME cells mediate changes in hepatic fibrosis during HCC progression. In this study, two pathways related to hepatic fibrosis signaling were enriched for DEGs: The HSC Activation Pathway and The Hepatic Fibrosis Signaling Pathway. A key molecular and cellular driver in the progression of liver fibrosis is the activation of HSCs. In this study, myofibroblast activation was observed as evidenced by increased ACTA2 in the TME. The increased expression of TME-derived ACTA2 is likely due to increased TGFB expression in the TME, which is activated from its latent form by integrin proteins. Interestingly, several integrins were upregulated in the HCC tumors, suggesting that HCC tumor cells are actively promoting TGFB-mediated activation of myofibroblasts. In addition to increased ACTA2 expression, the presence of activated myofibroblasts is further evidenced by upregulation of 21 type I and type III collagen genes in the TME. Type I and type III collagen represent the most abundant types of collagen found in fibrotic/cirrhotic liver tissues (Rojkind et al., 1979; Aycock and Seyer, 2009; Decaris et al., 2015), and activated myofibroblasts are known to be one of the greatest producers of these collagen types during liver fibrosis deposition (Shang et al., 2018). Together, the combined expression of TME-derived (TGFB and IGF1) and HCC-tumor derived (TGFA) upstream regulators of HSC activation suggests both the HCC tumor cells and TME cells are promoting collagen deposition.

In addition to evidence of collagen deposition, differential expression of genes involved in ECM remodeling was also observed. MMPs and TIMPs are major players in ECM remodeling and play a role in HCC progression and invasion (Scheau et al., 2019). In this study, overexpression of MMP13 and TIMP1 was observed in the HCC tumor cells. The increased 

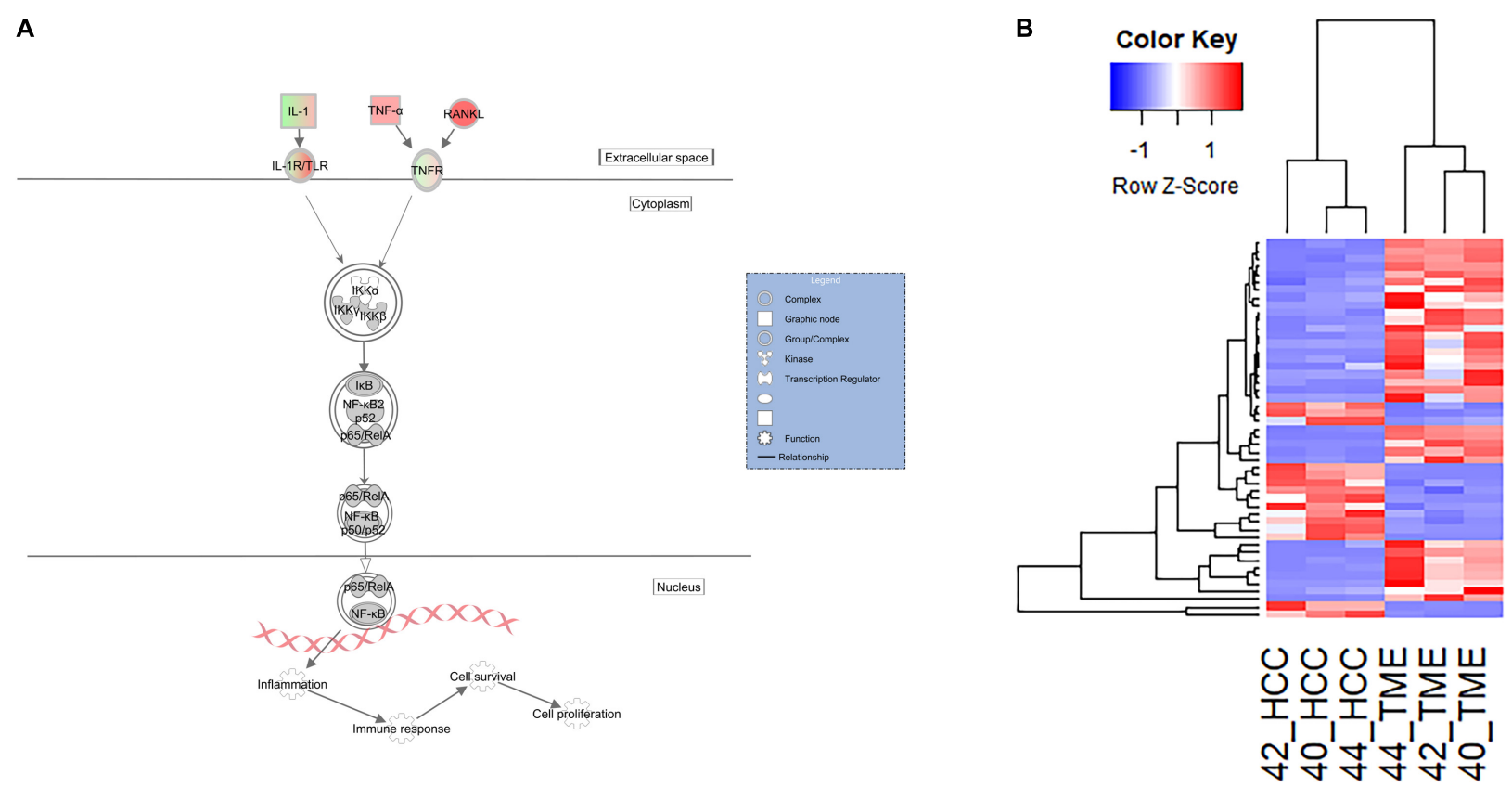

FIGURE 5 | Differential Expression of Genes Inolved in the Innate Immune and Inflammatory System. (A) Differential expression within the NF- $\kappa$ B canonical pathway in IPA (edited for simpler visualization; for full pathway, see Supplementary Figure 3). Molecule interactions are shown as explained in the legend. DEGs are highlighted either in red (upregulated TME genes) or green (upregulated HCC tumor genes). Color intensity indicates Increasing or decreasing degree of fold change. (B) Heatmap of the 49 genes differentially expressed in the NF-kB signaling pathway for all 6 samples.

expression was linked to TME-derived TGFB and TNF, as both TGFB and TNF are known to be potent inducers of MMP13 (Leivonen et al., 2002; Liacini et al., 2003), while TNF is known to modulate TIMP1 expression ( $\mathrm{Li}$ et al., 1999; Nee et al., 2004). Additionally, HCC derived ILIA and TGFA appear to be promoting upregulation of TME-derived $M M P 9$ and $M M P 2$, respectively. TIMP2 and TIMP1 are specific inhibitors of $M M P 2$ and MMP9, respectively (Chen et al., 2020). MMP2 degrades ECM components of the basement membrane and facilitates tumor invasion while TIMP2 regulates $M M P 2$ activity. In the context of clinical observations in HCC patients, several studies have revealed that an imbalance of MMP2 and TIMP2 expression is associated with poorer prognosis of HCC (Giannelli et al., 2002; Daniele et al., 2014). As both MMP2 and TIMP2 are expressed at high levels in the TME of this study, these results suggest that the clinically observed imbalance in MMP2 and TIMP2 expression is likely mediated by the TME as opposed to the HCC cells themselves. Like MMP2, MMP9 also degrades ECM components of the basement membrane to help facilitate tumor invasion while TIMP1 regulates MMP9 activity. Clinical observations have shown that higher expression of MMP9 is associated with capsular HCC infiltration (Arii et al., 1996). The increased MMP9 expression observed in the TME of this study suggests this clinical observation is also mediated by the TME as opposed to the HCC cells, although further studies are required to confirm these results.

In addition to hepatic fibrosis induction via myofibroblast activation and ECM remodeling, inflammation plays an essential role in mediating hepatic fibrosis and HCC progression. It has been extensively shown that chronic liver inflammation is associated with fibrosis and progression of HCC (Marra and Tacke, 2014; Tsuchida and Friedman, 2017; Ringelhan et al., 2018). In this study, evidence of TME-derived TNF binding to its receptor TNFRSF1B on HCC tumor cells was observed, resulting in upregulation of HCC-derived CCN2, MIP2, and VCAM1. These three genes play a role in recruiting macrophages and other leukocytes to sites of inflammation. In addition, LBP derived from the TME was also found to promote expression of HCC-derived MCP1 and VCAM1, both of which are involved in monocyte recruitment. This suggests that the HCC tumor cells are producing the necessary factors to promote local inflammation rather than TME cells responding to the abnormal cell growth. However, as this study was performed in SCID mice with compromised immune responses, the lack of signaling in the TME could also be due to the weakened immune system in this model. Therefore, further studies using immunocompetent model systems are required to further investigate the role of the TME in leukocyte recruitment. In addition to the hepatic fibrosis pathway, the NF- $\mathrm{B}$ signaling pathway was also significantly activated in the TME in this study. NF- $\kappa B$ is a key transcriptional regulator of inflammatory responses and plays a prominent role in chronic liver diseases (Luedde and Schwabe, 2011). Although this pathway was significantly activated in the TME, differential expression of NF- $\mathrm{B}$ target genes was not observed. This is likely due to binding of HCCderived IL1A to TME-derived IL1R2, a decoy receptor that binds IL1A to prevent subsequent signal transduction. This result suggests that the TME is expressing IL1R2 in order to limit an 

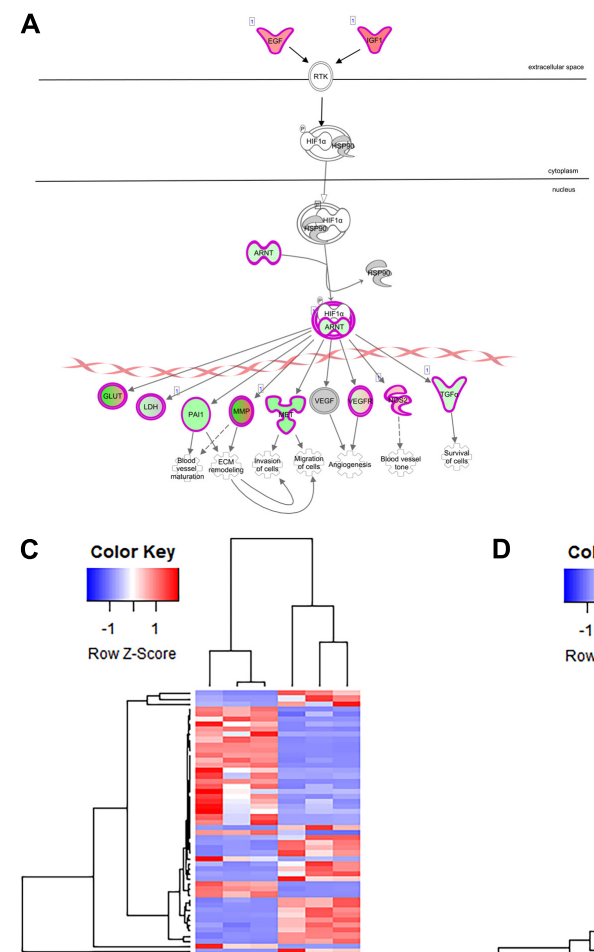

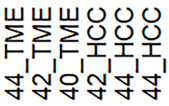

D

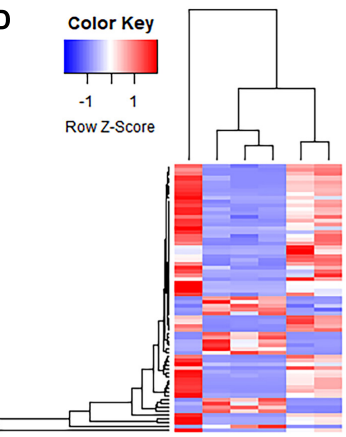

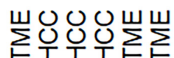

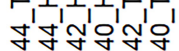

B

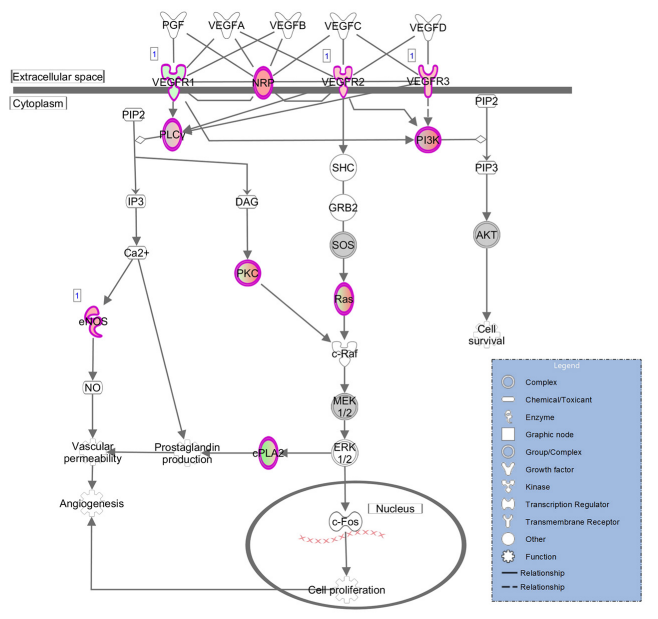

FIGURE 6 | Differential Expression of Genes Involved in Neo angiogenesis. Differential expression within the (A) HIF1 $\alpha$ Signaling canonical pathway and (B) VEGF Family Ligand-Receptor Interactions canonical pathway in IPA. Both pathways are edited for simpler visualization (for full pathway, see Supplementary

Figures 4, 5). Molecule interactions are shown as explained in the legend. DEGs are highlighted either in red (upregulated TME genes) or green (upregulated HCC tumor genes). Color intensity indicates increasing or decreasing degree of fold change. (C) Heatmap of the 51 DEGs in the HIF1 $\alpha$ Signaling pathway for all 6 samples. (D) Heatmap of the 20 DEGs in the VEGF Family Ligand-Receptor Interactions pathway for all 6 samples.

overactive inflammatory and immune response, providing an immunotolerant microenvironment for the HCC tumors.

HCC tumors in general are characterized as highly vascularized and hypoxic tumors (Yang and Poon, 2008; Chen and Lou, 2017). However, the mechanisms through which HCC tumors become vascularized and hypoxic are not well understood. In this study, two pathways related to angiogenesis were enriched for DEGs and activated in the TME: the HIF $1 \alpha$ signaling pathway and the VEGF family ligandreceptor interactions pathway. In the Hif $1 \alpha$ signaling pathway, TME-derived growth factors IGF and EGF are likely promoting HCC-tumor derived $A R N T$, which is in turn promoting the expression of known pro-angiogenesis genes PAI1, and TGFA. Studies have shown that PAI1 is upregulated in several cancers and represents a major contributor to angiogenesis in many cancers including HCC (Isogai et al., 2001; Geis et al., 2015). As described above, TGFA was upregulated in the HCC tumors acting as an upstream regulator of hepatic fibrosis. TGFA is also a pro-angiogenic factor whose expression is induced via activation of the HIF1 $\alpha$ signaling pathway, and has been shown to be a potent angiogenic factor in a variety of tumors (Schreiber et al., 1986). Although studies have shown overexpression of TGFA in HCC preclinical models and in clinical studies (Tomiya and Fujiwara, 1996; Jaiswal, 2014), there is limited knowledge on the angiogenic effect of TGFA specifically in HCC tumors. Our data suggests that TGFA could be stimulating HCC growth and fibrosis via its angiogenic potential.

With regards to the VEGF family ligand-receptor interactions pathway, VEGFR1 and VEGFR2 are high-affinity receptors for angiogenesis factors and have been found to play key autocrine signaling roles in HCC cell proliferation (de Vries et al., 1992; Quinn et al., 1993; Peng et al., 2014). When bound to their ligands, predominantly VEGF, downstream signaling leads to promoting vascular permeability and endothelial cell proliferation. VEGFR1 plays critical roles in promoting pathological tumor angiogenesis and invasion (Ceci et al., 2020), and HCC tumor cells have been shown to express VEGFR1 resulting in induction of autocrine VEGF signaling (Peng et al., 2014). VEGFR2 expression regulates vascular endothelial cell function by promoting proliferation and survival (Holmes et al., 2007), while NRP1 is a pro-angiogenic co-receptor and has been shown to be expressed in both HCC tumor cells and hepatic endothelial cells (Bergé et al., 2011). Therefore, TME-derived VEGFR2 and NRP1 is likely being expressed by endothelial 
cells surrounding the HCC tumor in order to promote a new vascular network for the xenograft. As differential expression of ligands that bind to any of the VEGFRs was not observed, it is uncertain whether VEGF signaling is actually occurring at this point in the tumorigenic process, although previous studies have demonstrated the hypervascular nature of Oncopig HCC xenografts (Schachtschneider et al., 2017a). However, HCC tumor and TME-derived expression of several VEGFRs demonstrates that the HCC tumor and TME cells are displaying critical receptors for promotion of angiogenesis.

In conclusion, this study investigated gene expression profiles in the Oncopig HCC tumor xenograft model, identifying crosstalk between HCC tumors and the TME related to HCC progression, including hepatic fibrosis, inflammation, and angiogenesis. We revealed multiple pathways and specific gene interactions between HCC tumors and the TME that provide insights into how HCC tumor and TME cells cooperate to promote HCC proliferation. However, several limitations were identified in this study. First, the small sample size used limits our ability to control for variability and identify outliers, and therefore larger further confirmatory studies are required. The small sample size also limited our ability to investigate the possible gender-based stratification of samples observed on principal component 2. Second, although TP53 driver mutations are commonly observed in clinical HCC, the KRAS driver mutation is rarely observed in HCC (Hussain et al., 2007; Turhal et al., 2015). However, previous publications have demonstrated that Oncopig HCC recapitulates human HCC in terms of transcriptional profiles, histological phenotypes, AFP expression, proliferation rates, migration rates, and chemotherapeutic susceptibilities (Schachtschneider et al., 2017a; Gaba et al., 2020). Therefore, despite the presence of a KRAS driver mutation, the Oncopig HCC model represents a clinically relevant large animal HCC model. Additionally, while utilizing RNA-seq data allows for genome-wide profiling of all expressed genes, the crossspecies analysis performed in this study limited the analysis to one-to-one orthologues. As the final one-to-one orthologue set used in this study represents $25.5 \%$ and $44.4 \%$ of genes annotated in the mouse and pig genome, respectively, our ability to fully elucidate all the pathway disruptions and HCC-TME crosstalk is limited. In addition, as RNA-seq only allows for quantification of gene expression at the mRNA level, further studies are required to investigate the impact of protein-protein interactions and posttranslational modifications on HCC tumor development, as well as confirm the results of the pathway analysis presented here. Finally, as the presence of tumor infiltrating lymphocytes and

\section{REFERENCES}

Akahoshi, K., Tanaka, S., Mogushi, K., Shimada, S., Matsumura, S., Akiyama, Y., et al. (2016). Expression of connective tissue growth factor in the livers of non-viral hepatocellular carcinoma patients with metabolic risk factors. J. Gastroenterol. 51, 910-922. doi: 10.1007/s00535-015-1159-8

Arii, S., Mise, M., Harada, T., Furutani, M., Ishigami, S. I., Niwano, M., et al. (1996). Overexpression of matrix metalloproteinase 9 gene in hepatocellular carcinoma with invasive potential. Hepatology 24, 316-322. doi: 10.1053/jhep.1996.v24. pm0008690399 other immune cells is a key component of the TME, our inability to fully characterize signaling originating from these cell types represents a limitation of this and other studies using SCID mice for xenograft development. Therefore, while this model allows for efficient bioinformatics-based read separation to assess HCC and TME cross talk, elucidation of the role the immune system plays could not be determined.

\section{DATA AVAILABILITY STATEMENT}

The datasets presented in this study can be found in online repositories. The names of the repository/repositories and accession number(s) can be found below: https://www.ncbi.nlm. nih.gov/sra, PRJNA685801.

\section{ETHICS STATEMENT}

The animal study was reviewed and approved by University of Illinois at Urbana-Champaign IACUC.

\section{AUTHOR CONTRIBUTIONS}

KS, ME-K, LS, and RG conceptualized and designed the study. SP, $\mathrm{KS}$, and AS performed the experiments. SP and AS performed the data analysis. SP wrote the manuscript. All authors contributed to the discussion of results and manuscript corrections.

\section{FUNDING}

This work was supported by the National Institutes of Health National Cancer Institute (1R21CA219461-01A1), United States Department of Defense (Translational Team Science Award CA150590), the Cooperative Research Program for Agriculture Science \& Technology Development (PJ009103) of the Rural Development Administration, Republic of Korea, and the Department of Radiology, University of Illinois at Chicago.

\section{SUPPLEMENTARY MATERIAL}

The Supplementary Material for this article can be found online at: https://www.frontiersin.org/articles/10.3389/fgene. 2021.657330/full\#supplementary-material

Aycock, R. S., and Seyer, J. M. (2009). Collagens of normal and cirrhotic human liver. Connect Tissue Res. 23, 19-31. doi: 10.3109/03008208909103901

Baghban, R., Roshangar, L., Jahanban-Esfahlan, R., Seidi, K., Ebrahimi-Kalan, A., Jaymand, M., et al. (2020). Tumor microenvironment complexity and therapeutic implications at a glance. Cell Commun. Signal. 18, 1-19. doi: 10. 1186/s12964-020-0530-4

Bergé, M., Allanic, D., Bonnin, P., De Montrion, C., Richard, J., Suc, M., et al. (2011). Neuropilin-1 is upregulated in hepatocellular carcinoma and contributes to tumour growth and vascular remodelling. J. Hepatol. 55, 866875. doi: 10.1016/j.jhep.2011.01.033 
Breton, J. D., Heydet, D., Starrs, L. M., Veldre, T., and Ghildyal, R. (2018). Molecular changes during TGF $\beta$-mediated lung fibroblast-myofibroblast differentiation: implication for glucocorticoid resistance. Physiol. Rep. 6, 1-12. doi: 10.14814/phy2.13669

Ceci, C., Atzori, M. G., Lacal, P. M., and Graziani, G. (2020). Role of VEGFs/VEGFR-1 signaling and its inhibition in modulating tumor invasion: experimental evidence in different metastatic cancer models. Int. J. Mol. Sci. 21, 1-53. doi: 10.3390/ijms21041388

Chen, C., and Lou, T. (2017). Hypoxia inducible factors in hepatocellular carcinoma. Oncotarget 8, 46691-46703. doi: 10.18632/oncotarget. 17358

Chen, G., Ge, D., Zhu, B., Shi, H., and Ma, Q. (2020). Upregulation of matrix metalloproteinase 9 (MMP9)/tissue inhibitor of metalloproteinase 1 (TIMP1) and MMP2/TIMP2 ratios may be involved in lipopolysaccharide-induced acute lung injury. J. Int. Med. Res. 48, 1-10. doi: 10.1177/0300060520919592

Conway, T., Wazny, J., Bromage, A., Tymms, M., Sooraj, D., Williams, E. D., et al. (2012). Xenome-a tool for classifying reads from xenograft samples. Bioinformatics 28, 172-178. doi: 10.1093/bioinformatics/bts236

Cook-Mills, J. M., Marchese, M. E., and Abdala-Valencia, H. (2011). Vascular cell adhesion molecule-1 expression and signaling during disease: regulation by reactive oxygen species and antioxidants. Antioxidants Redox Signal. 15, 1607-1638. doi: 10.1089/ars.2010.3522

Daniele, A., Divella, R., Quaranta, M., Mattioli, V., Casamassima, P., Paradiso, A., et al. (2014). Clinical and prognostic role of circulating MMP-2 and its inhibitor TIMP-2 in HCC patients prior to and after trans-hepatic arterial chemo-embolization. Clin. Biochem. 47, 184-190. doi: 10.1016/j.clinbiochem. 2013.11.022

de Vries, C., Escobedo, J. A., Ueno, H., Houck, K., Ferrara, N., and Williams, L. T. (1992). The fms-like tyrosine kinase, a receptor for vascular endothelial growth factor. Science 255, 989-991. doi: 10.1126/science.1312256

Decaris, M. L., Emson, C. L., Li, K., Gatmaitan, M., Luo, F., Cattin, J., et al. (2015). Turnover rates of hepatic collagen and circulating collagen-associated proteins in humans with chronic liver disease. PLoS One 10:e0123311. doi: 10.1371/journal.pone.0123311

Dobin, A., Davis, C. A., Schlesinger, F., Drenkow, J., Zaleski, C., Jha, S., et al. (2013). STAR: ultrafast universal RNA-seq aligner. Bioinformatics 29, 15-21. doi: 10.1093/bioinformatics/bts635

Flores, A., and Marrero, J. A. (2014). Emerging trends in hepatocellular carcinoma: Focus on diagnosis and therapeutics. Clin. Med. Insights Oncol. 8, 71-76. doi: 10.4137/CMO.S9926

Foissac, S., Djebali, S., Munyard, K., Vialaneix, N., Rau, A., Muret, K., et al. (2019). Multi-species annotation of transcriptome and chromatin structure in domesticated animals. BMC Biol. 17:108. doi: 10.1186/s12915-019-0726-5

Gaba, R. C., Elkhadragy, L., Edward Boas, F., Chaki, S., Chen, H. H., El-Kebir, M., et al. (2020). Development and comprehensive characterization of porcine hepatocellular carcinoma for translational liver cancer investigation. Oncotarget 11, 2686-2701. doi: 10.18632/oncotarget.27647

Gandhi, C. R. (2020). Pro- and anti-fibrogenic functions of gram-negative bacterial lipopolysaccharide in the liver. Front. Med. 7:130. doi: 10.3389/fmed.2020. 00130

Geis, T., Döring, C., Popp, R., Grossmann, N., Fleming, I., Hansmann, M. L., et al. (2015). HIF-2alpha-dependent PAI-1 induction contributes to angiogenesis in hepatocellular carcinoma. Exp. Cell Res. 331, 46-57. doi: 10.1016/j.yexcr.2014. 11.018

Germain, A. R. D. (2017). 乳鼠心肌提取 HHS public access. Physiol. Behav. 176, 139-148.

Giannelli, G., Bergamini, C., Marinosci, F., Fransvea, E., Quaranta, M., Lupo, L., et al. (2002). Clinical role of MMP-2/TIMP-2 imbalance in hepatocellular carcinoma. Int. J. Cancer 97, 425-431. doi: 10.1002/ijc.1635

Goodwin, A., and Jenkins, G. (2009). Role of integrin-mediated TGF $\beta$ activation in the pathogenesis of pulmonary fibrosis. Biochem. Soc. Trans. 37, 849-854. doi: 10.1042/BST0370849

Harada, K. I., Shiota, G., and Kawasaki, H. (1999). Transforming growth factor- $\alpha$ and epidermal growth factor receptor in chronic liver disease and hepatocellular carcinoma. Liver 19, 318-325. doi: 10.1111/j.1478-3231.1999.tb00056.x

Harris, W. T., Kelly, D. R., Zhou, Y., Wang, D., Macewen, M., Hagood, J. S., et al. (2013). Myofibroblast differentiation and enhanced Tgf-B signaling in cystic fibrosis lung disease. PLoS One 8:e0070196. doi: 10.1371/journal.pone.00 70196

Higashi, T., Friedman, S. L., and Hoshida, Y. (2017). Hepatic stellate cells as key target in liver fibrosis. Adv. Drug Deliv. Rev. 121, 27-42. doi: 10.1016/j.addr. 2017.05.007

Ho, J. W., Poon, R. T., Tong, C. S., and Fan, S. T. (2004). Clinical significance of serum vascular cell adhesion molecule-1 levels in patients with hepatocellular carcinoma. World J. Gastroenterol. 10, 2014-2018. doi: 10.3748/wjg.v10.i14. 2014

Holmes, K., Roberts, O. L., Thomas, A. M., and Cross, M. J. (2007). Vascular endothelial growth factor receptor-2: structure, function, intracellular signalling and therapeutic inhibition. Cell. Signal. 19, 2003-2012. doi: 10.1016/j.cellsig. 2007.05.013

Hussain, S. P., Schwank, J., Staib, F., Wang, X. W., and Harris, C. C. (2007). TP53 mutations and hepatocellular carcinoma: insights into the etiology and pathogenesis of liver cancer. Oncogene 26, 2166-2176. doi: 10.1038/sj.onc. 1210279

Isogai, C., Laug, W. E., Shimada, H., Declerck, P. J., Stins, M. F., Durden, D. L., et al. (2001). Plasminogen activator inhibitor-1 promotes angiogenesis by stimulating endothelial cell migration toward fibronectin. Cancer Res. 61, 5587-5594.

Jaiswal (2014). 基因的改变NIH public access. Bone 23, 1-7.

Khan, Z., and Marshall, J. F. (2016). The role of integrins in TGF $\beta$ activation in the tumour stroma. Cell Tissue Res. 365, 657-673. doi: 10.1007/s00441-016-2474-y

Kirstein, M. M. (2016). Epidemiology and risk factors of cholangiocarcinoma. Visc Med. 32, 395-400. doi: 10.1159/000453013

Krämer, A., Green, J., Pollard, J., and Tugendreich, S. (2014). Causal analysis approaches in ingenuity pathway analysis. Bioinformatics 30, 523-530. doi: 10.1093/bioinformatics/btt703

Leivonen, S. K., Chantry, A., Häkkinen, L., Han, J., and Kähäri, V. M. (2002). Smad 3 mediates transforming growth factor- $\beta$-induced collagenase- 3 (matrix metalloproteinase-13) expression in human gingival fibroblasts: evidence for cross-talk between Smad3 and p38 signaling pathways. J. Biol. Chem. 277, 46338-46346. doi: 10.1074/jbc.M206535200

Li, B., and Dewey, C. N. (2011). RSEM: accurate transcript quantification from RNA-Seq data with or without a reference genome. BMC Bioinformatics 12:323. doi: 10.1186/1471-2105-12-323

Li, Y. Y., McTiernan, C. F., and Feldman, A. M. (1999). Proinflammatory cytokines regulate tissue inhibitors of metalloproteinases and disintegrin metalloproteinase in cardiac cells. Cardiovasc. Res. 42, 162-172. doi: 10.1016/ S0008-6363(98)00297-1

Liacini, A., Sylvester, J., Li, W. Q., Huang, W., Dehnade, F., Ahmad, M., et al. (2003). Induction of matrix metalloproteinase-13 gene expression by TNF- $\alpha$ is mediated by MAP kinases, AP-1, and NF- $\mathrm{B}$ transcription factors in articular chondrocytes. Exp. Cell Res. 288, 208-217. doi: 10.1016/S0014-4827(03) 00180-0

Liu, T., Zhang, L., Joo, D., and Sun, S. C. (2017). NF-кB signaling in inflammation. Signal Transduct. Target. Ther. 2:17023. doi: 10.1038/sigtrans.2017.23

Liu, X. L., Li, F. Q., Liu, L. X., Li, B., and Zhou, Z. P. (2013). TNF- $\alpha$, HGF and macrophage in peritumoural liver tissue relate to major risk factors of HCC recurrence. Hepatogastroenterology 60, 1121-1126. doi: 10.5754/hge12982

Luedde, T., and Schwabe, R. F. (2011). NF-[kappa]B in the liver[mdash]linking injury, fibrosis and hepatocellular carcinoma. Nat. Rev. Gastroenterol. Hepatol. 8, 108-118. doi: 10.1038/nrgastro.2010.213

Makino, Y., Hikita, H., Kodama, T., Shigekawa, M., Yamada, R., Sakamori, R., et al. (2018). CTGF mediates tumor-stroma interactions between hepatoma cells and hepatic stellate cells to accelerate HCC progression. Cancer Res. 78, 4902-4914. doi: 10.1158/0008-5472.CAN-17-3844

Marra, F., and Tacke, F. (2014). Roles for chemokines in liver disease. Gastroenterology 147, 577.e1-594.e1. doi: 10.1053/j.gastro.2014.06.043

Mazzocca, A., Fransvea, E., Dituri, F., Lupo, L., Antonaci, S., and Giannelli, G. (2010). Down-regulation of connective tissue growth factor by inhibition of transforming growth factor $\beta$ blocks the tumor-stroma cross-talk and tumor progression in hepatocellular carcinoma. Hepatology 51, 523-534. doi: 10.1002/ hep. 23285

Mhatre, V. H., Ji-Ann, L., and Kcm. (2012). 基因的改变 NIH public access. Bone $23,1-7$. 
Moles, A., Murphy, L., Wilson, C. L., Chakraborty, J. B., Fox, C., Park, E. J., et al. (2014). A TLR2/S100A9/CXCL-2 signaling network is necessary for neutrophil recruitment in acute and chronic liver injury in the mouse. J. Hepatol. 60, 782-791. doi: 10.1016/j.jhep.2013.12.005

Mora-Buch, R., Dotti, I., Planell, N., Calderón-Gómez, E., Jung, P., Masamunt, M. C., et al. (2016). Epithelial IL-1R2 acts as a homeostatic regulator during remission of ulcerative colitis. Mucosal Immunol. 9, 950-959. doi: 10.1038/mi. 2015.108

Nakerakanti, S. S., Bujor, A. M., and Trojanowska, M. (2011). CCN2 is required for the TGF- $\beta$ induced activation of Smad1 - Erk1/2 signaling network. PLoS One 6:e0021911. doi: 10.1371/journal.pone.0021911

Nee, L. E., McMorrow, T., Campbell, E., Slattery, C., and Ryan, M. P. (2004). TNF$\alpha$ and IL-1 $\beta$-mediated regulation of MMP-9 and TIMP-1 in renal proximal tubular cells. Kidney Int. 66, 1376-1386. doi: 10.1111/j.1523-1755.2004.00900.x

Osborn, L., Hession, C., Tizard, R., Vassallo, C., Luhowskyj, S., Chi-Rosso, G., et al. (1989). Direct expression cloning of vascular cell adhesion molecule 1, a cytokine-induced endothelial protein that binds to lymphocytes. Cell 59, 1203-1211. doi: 10.1016/0092-8674(89)90775-7

Peng, S., Wang, Y., Peng, H., Chen, D., Shen, S., Peng, B., et al. (2014). Autocrine vascular endothelial growth factor signaling promotes cell proliferation and modulates sorafenib treatment efficacy in hepatocellular carcinoma. Hepatology 60, 1264-1277. doi: 10.1002/hep.27236

Puche, J. E., Saiman, Y., and Friedman, S. L. (2013). Hepatic stellate cells and liver fibrosis. Compr. Physiol. 3, 1473-1492. doi: 10.1002/cphy.c120035

Qin, C. C., Liu, Y. N., Hu, Y., Yang, Y., and Chen, Z. (2017). Macrophage inflammatory protein-2 as mediator of inflammation in acute liver injury. World J. Gastroenterol. 23, 3043-3052. doi: 10.3748/wjg.v23.i17.3043

Quinn, T. P., Peters, K. G., De Vries, C., Ferrara, N., and Williams, L. T. (1993). Fetal liver kinase 1 is a receptor for vascular endothelial growth factor and is selectively expressed in vascular endothelium. Proc. Natl. Acad. Sci. U.S.A. 90, 7533-7537. doi: 10.1073/pnas.90.16.7533

Rahib, L., Smith, B. D., Aizenberg, R., Rosenzweig, A. B., Fleshman, J. M., and Matrisian, L. M. (2020). Projecting cancer incidence and deaths to 2030: the unexpected burden of thyroid, liver, and pancreas cancers in the united states. Cancer Res. 74, 2913-2921. doi: 10.1158/0008-5472.CAN-14-0155

Ringelhan, M., Pfister, D., O’Connor, T., Pikarsky, E., and Heikenwalder, M. (2018). The immunology of hepatocellular carcinoma review-article. Nat. Immunol. 19, 222-232. doi: 10.1038/s41590-018-0044-z

Rojkind, M., Giambrone, M., and Biempica, L. (1979). collagen types in normal and cirrhotic liver. Gastroenterology 76, 710-719. doi: 10.1016/S0016-5085(79) 80170-5

Schachtschneider, K. M., Schwind, R. M., Darfour-Oduro, K. A., De, A. K., Rund, L. A., Singh, K., et al. (2017a). A validated, transitional and translational porcine model of hepatocellular carcinoma. Oncotarget 8, 63620-63634. doi: 10.18632/ oncotarget.18872

Schachtschneider, K. M., Schwind, R. M., and Newson, J. (2017b). The oncopig cancer model: an innovative large animal translational oncology platform. Front. Oncol. 7:190. doi: 10.3389/fonc.2017.00190

Scheau, C., Badarau, I. A., Costache, R., Caruntu, C., Mihai, G. L., Didilescu, A. C., et al. (2019). The role of matrix metalloproteinases in the epithelialmesenchymal transition of hepatocellular carcinoma. Anal. Cell. Pathol. 2019:9423907. doi: 10.1155/2019/9423907

Schook, L. B., Collares, T. V., Hu, W., and Liang, Y. (2015). A genetic porcine model of cancer. PLoS One 10:e0128864. doi: 10.1371/journal.pone.0128864

Schreiber, A. B., Winkler, M. E., Derynck, R., Science, S., Series, N., Jun, N., et al. (1986). Transforming growth Factor- $\alpha$ : a more potent angiogenic mediator than epidermal growth factor. Science 232, 1250-1253.

Shang, L., Hosseini, M., Liu, X., Kisseleva, T., and Brenner, D. A. (2018). Human hepatic stellate cell isolation and characterization. J. Gastroenterol. 53, 6-17. doi: 10.1007/s00535-017-1404-4

Song, F. N., Duan, M., Liu, L. Z., Wang, Z. C., Shi, J. Y., Yang, L. X., et al. (2014). RANKL promotes migration and invasion of hepatocellular carcinoma cells via NF-кb-mediated epithelial-mesenchymal transition. PLoS One 9:e0108507. doi: 10.1371/journal.pone.0108507

Steinhoff, G., Behrend, M., Schrader, B., Duijvestijn, A. M., and Wonigeit, K. (1993). Expression patterns of leukocyte adhesion ligand molecules on human liver endothelia: lack of ELAM-1 and CD62 inducibility on sinusoidal endothelia and distinct distribution of VCAM-1, ICAM-1, ICAM-2, and LFA-3. Am. J. Pathol. 142, 481-488.

Tan, W., Luo, X., Li, W., Zhong, J., Cao, J., Zhu, S., et al. (2019). TNF- $\alpha$ is a potential therapeutic target to overcome sorafenib resistance in hepatocellular carcinoma. EBioMedicine 40, 446-456. doi: 10.1016/j.ebiom.2018.12.047

Tanimoto, K. (2000). Mechanism of regulation of the hypoxia-inducible factorlalpha by the von Hippel-Lindau tumor suppressor protein. EMBO J. 19, 4298-4309. doi: 10.1093/emboj/19.16.4298

Tomiya, T., and Fujiwara, K. (1996). Serum transforming growth factor $\alpha$ level as a marker of hepatocellular carcinoma complicating cirrhosis. Cancer 77, 1056-1060.

Tsuchida, T., and Friedman, S. L. (2017). Mechanisms of hepatic stellate cell activation. Nat. Rev. Gastroenterol. Hepatol 14, 397-411. doi: 10.1038/nrgastro. 2017.38

Turhal, N. S., Savaş, B., Çoşkun, Ö, Baş, E., Karabulut, B., Nart, D., et al. (2015). Prevalence of K-Ras mutations in hepatocellular carcinoma: a turkish oncology group pilot study. Mol. Clin. Oncol. 3, 1275-1279. doi: 10.3892/mco.20 15.633

Warr, A., Affara, N., Aken, B., Beiki, H., Bickhart, D. M., Billis, K., et al. (2020). An improved pig reference genome sequence to enable pig genetics and genomics research. Gigascience 9:giaa051. doi: 10.1093/gigascience/giaa051

Watsky, M. A., Weber, K. T., Sun, Y., and Postlethwaite, A. (2010). New Insights Into the Mechanism of Fibroblast to Myofibroblast Transformation and Associated Pathologies, 1st Edn. Amsterdam: Elsevier Inc, doi: 10.1016/S19376448(10)82004-0

Weir, L., Robertson, D., Leigh, I. M., Vass, J. K., and Panteleyev, A. A. (2011). Hypoxia-mediated control of HIF/ARNT machinery in epidermal keratinocytes. Biochim. Biophys. Acta Mol. Cell Res. 1813, 60-72. doi: 10.1016/j. bbamcr.2010.11.014

Whiteside, T. L. (2008). The tumor microenvironment and its role in promoting tumor growth. Oncogene 27, 5904-5912. doi: 10.1038/onc.2008.271

Yang, Z. F., and Poon, R. T. P. (2008). Vascular changes in hepatocellular carcinoma. Anat. Rec. 291, 721-734. doi: 10.1002/ar. 20668

Yeh, M. M., Larson, A. M., Campbell, J. S., Fausto, N., Rulyak, S. J., and Swanson, P. E. (2007). The expression of transforming growth factor- $\alpha$ in cirrhosis, dysplastic nodules, and hepatocellular carcinoma: an immunohistochemical study of 70 cases. Am. J. Surg. Pathol. 31, 681-689. doi: 10.1097/PAS. 0b013e31802ff7aa

Zhang, C. Y., Yuan, W. G., He, P., Lei, J. H., and Wang, C. X. (2016). Liver fibrosis and hepatic stellate cells: etiology, pathological hallmarks and therapeutic targets. World J. Gastroenterol. 22, 10512-10522. doi: 10.3748/wjg.v22.i48. 10512

Conflict of Interest: RG receives research funding from the National Insitutes of Health, the United States Department of Defense, Guerbet United States LLC, and Janssen Research Development LLC. KS receives research funding from the National Insitutes of Health, Guerbet United States LLC, and Janssen Research Development LLC. RG, KS, and LS are scientific consultants for Sus Clinicals, Inc.

Copyright (c) 2021 Patel, Sandur, El-Kebir, Gaba, Schook and Schachtschneider. This is an open-access article distributed under the terms of the Creative Commons Attribution License (CC BY). The use, distribution or reproduction in other forums is permitted, provided the original author(s) and the copyright owner(s) are credited and that the original publication in this journal is cited, in accordance with accepted academic practice. No use, distribution or reproduction is permitted which does not comply with these terms. 